NOTA

\title{
Una enorme congregación de Episcada hymenaea (Lepidoptera: Nymphalidae: Danainae) en las yungas del norte de Salta, Argentina
}

A Huge Aggregation of Episcada hymenaea (Lepidoptera: Nymphalidae: Danainae) in the Yungas of Northern Salta, Argentina

D.0.I.: https://doi.org/10.30550/j.azl/2018.62.2/5

\section{Quinteros, Ramón ${ }^{1}$; Ezequiel Núñez Bustos ${ }^{2 *}$}

1 Fragata Libertad 370 (4568), Barrio Armada Argentina, Profesor Salvador Mazza, Provincia de Salta, Argentina.

2 Colección de Lepidoptera Laboratorio Barcode, Museo Argentino de Ciencias Naturales "Bernardino Rivadavia", Av. Angel Gallardo 470, (1405) Ciudad Autónoma de Buenos Aires, Argentina.

argentinebutterflies@hotmail.com; ezequielnb@gmail.com

Resumen - Este trabajo documenta el primer registro de una gran congregación invernal de miles de ejemplares de Episcada hymenaea desde agosto de 2014 a octubre de 2017 en las Yungas del extremo norte de Argentina. Esta información fue documentada a través de fotografías y grabada en video y es comparada con la disponible en Brasil y Paraguay para la misma tribu. Mazza.

Palabras clave: Mariposas, Comportamiento gregario, Estación seca, Profesor Salvador

> Abstract - This work documents the first record of a large winter concentration of thousands of Episcada hymenaea specimens from August 2014 to October 2017 in the Yungas of the far north of Argentina. This information was documented through photographs and video recordings, and is compared to the available in Brazil and Paraguay for the same tribe.

Keywords: Butterflies, Gregarious behaviour, Dry season, Profesor Salvador Mazza.

\section{INTRODUCCIÓN}

Es relativamente conocido el hecho de que varias especies de la tribu Ithomiini forman congregaciones o agregaciones de alta densidad durante la estación seca o en el invierno del hemisferio sur sudamericano (entre junio y octubre), en el interior de bosques y selvas húmedos cercanos a cursos de agua con el supuesto objeto de protegerse del frío y/o del clima seco. Las mismas son denominadas en inglés aggregations o ithomiine pockets y en Brasil bolsâo de ithomiine (Brown y Vasconcellos-Neto, 1976; Freitas, Vasconcellos-Neto, Vanini, Trigo, Brown, 2001; Pinheiro, Medri, Salcedo, 2008). En la jurisdicción de la localidad fronteriza de

\footnotetext{
> Ref. bibliográfica: Quinteros, R.; Núñez Bustos, E. 2018. Una enorme congregación de Episcada hymenaea (Lepidoptera: Nymphalidae: Danainae) en las yungas del norte de Salta, Argentina. Acta zoológica lilloana 62 (2): 44-50.

> Recibido: 16/08/18 - Aceptado: 08/10/18

> URL de la revista: http://actazoologica.lillo.org.ar

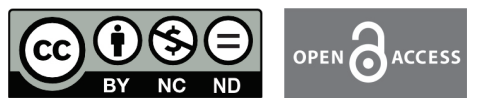

> Algunos derechos reservados. Esta obra está bajo una Licencia Creative Commons Atribución - No Comercial - Sin Obra Derivada 4.0 Internacional.
} 
Profesor Salvador Mazza (Departamento General José de San Martín, Salta), la mariposa llamada vulgarmente Translúcida Común (Episcada hymenaea hymenaea) (Fig. 1), es vista con frecuencia en jardines, en espacios del monte chaqueño o en zonas de las sierras de Tartagal que, al ingresar en Bolivia se convierten en las sierras del Aguaragüe (departamento Tarija) y que integran las últimas estribaciones del cordón montañoso de las sierras subandinas (yungas) en ese país. En Sudamérica se la halla en Bolivia, Brasil, Paraguay, Uruguay y Argentina en ambientes boscosos húmedos pero también modificados e incluso áreas urbanas. Se trata de la especie más común del género en Argentina de la tribu Ithomiini y la de más amplia distribución geográfica, la cual cubre todo el norte y centro del país y alcanza hasta las provincias de Buenos Aires, La Pampa y Mendoza (Hayward, 1973; Núñez Bustos, 2010; Volkmann y Núñez Bustos, 2013). En las yungas del noroeste argentino es una de las más comunes especies de la tribu y la única perteneciente al género Episcada en dicha región.

El clima de la zona es tropical serrano con estación seca. Las precipitaciones pueden alcanzar a $2000 \mathrm{~mm}$ anuales y ocurren entre noviembre y marzo. Las temperaturas en verano son altas y pueden alcanzar los $50^{\circ} \mathrm{C}$. El invierno es seco, posee gran amplitud térmica diaria y pueden haber heladas. La vegetación es la correspondiente a la zona basal de las yungas, hallándose entre los árboles Lapacho rosado (Handroanthus impetiginosus), Roble criollo (Ambureana cearensis), Cebil (Anadenathera colubrina), Pacará (Enterolobium contortosiliquum), Guabiyú (Eugenia pungens), Tusca (Vachellia aroma), etc. También hay algunas especies exóticas invasoras como Papaya (Carica papaya), Banano (Musa x paradisiaca), Tártago (Ricinus communis), Guayaba (Psidium

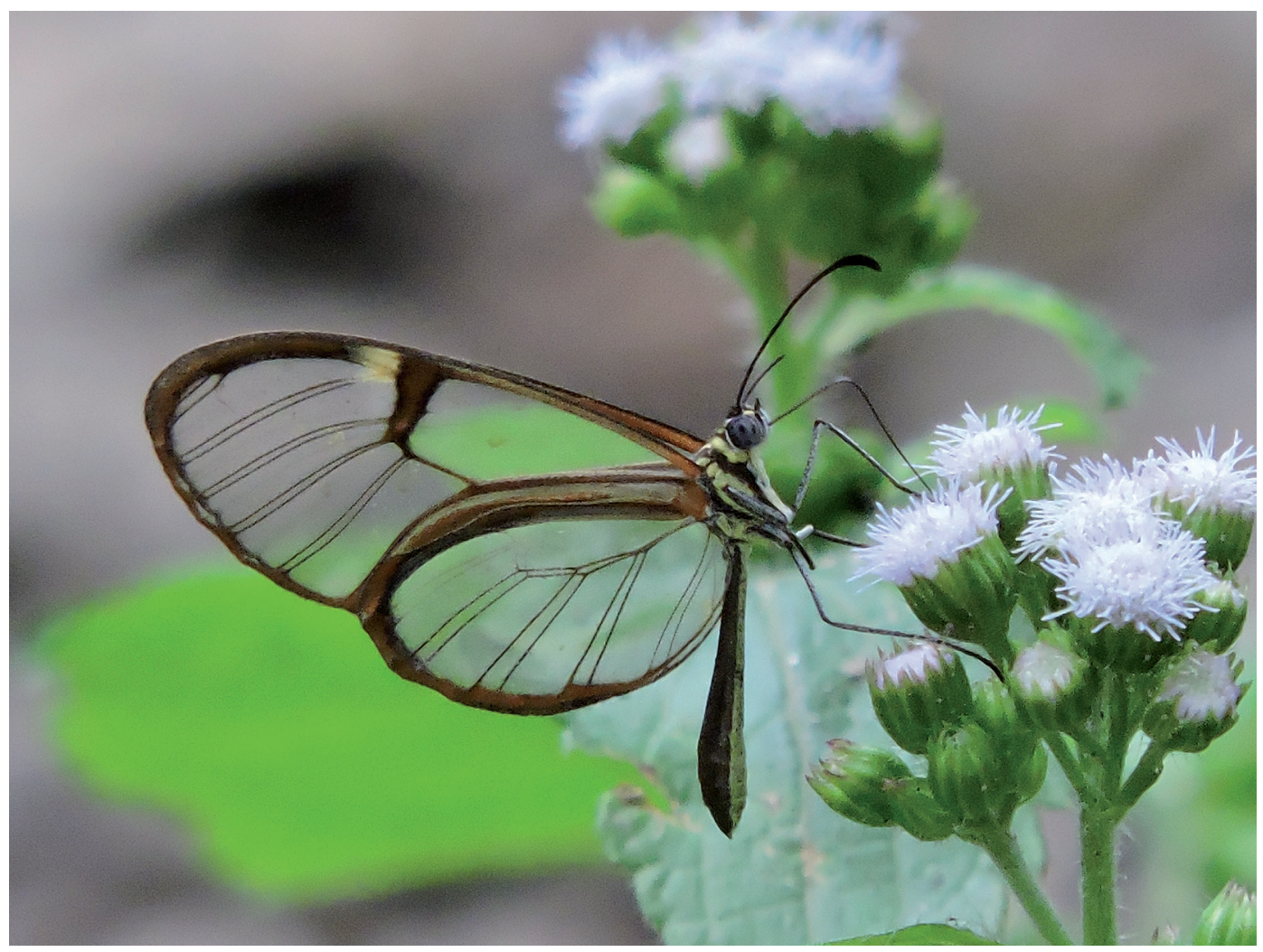

Fig. 1. Episcada hymenaea hymenaea. Foto: Ramón Quinteros. 18/04/2015. 
guajaba), Urucú (Bixia orellana) y Cítricos (Citrus spp.).

Las observaciones, fotografías y grabaciones fueron realizadas por RQ en un arroyo del Paraje la Toma, situado al oeste de la ciudad (2203'03"S - 63० 43'01'O). El arroyo es un pequeño curso de agua permanente que baja de casi 900 msnm encajonado en altas paredes cubiertas de densa vegetación de la sierra (Fig. 2). Cuando llueve acumula las aguas desde las alturas y varias vertientes, descargando todo su caudal en el arroyo Pocitos, ubicado a $3 \mathrm{~km}$. Este fenómeno constantemente erosiona las laderas y el lecho de la quebrada. Posee un microclima especial, pero es un ambiente en constante degradación por acción del hombre, de la naturaleza misma y de los incendios espontáneos muy recurrentes en la estación seca.

\section{DESCRIPCIÓN DEL REGISTRO}

El día 24 de agosto de 2014, después del mediodía y caminando por el paraje La Toma (cercano a dos $\mathrm{Km}$ de la ciudad de Profesor Salvador Mazza), RQ se encontró, en el sector occidental de la sierra y resguardado por una espesa vegetación compuesta de árboles, arbustos y helechos, una enorme congregación de miles de individuos de Episcada hymenaea (Fig. 3). La mayor parte del tiempo permanecieron posadas siempre con la cabeza hacia arriba y levemente inclinadas en grandes grupos, adheridas en arbustos, troncos, raíces, en el suelo cubierto de hojas, en rocas y paredes occidentales del cerro, húmedas y cubiertas de musgos. De esa manera no quedaban expuestas en forma directa a los rayos solares.

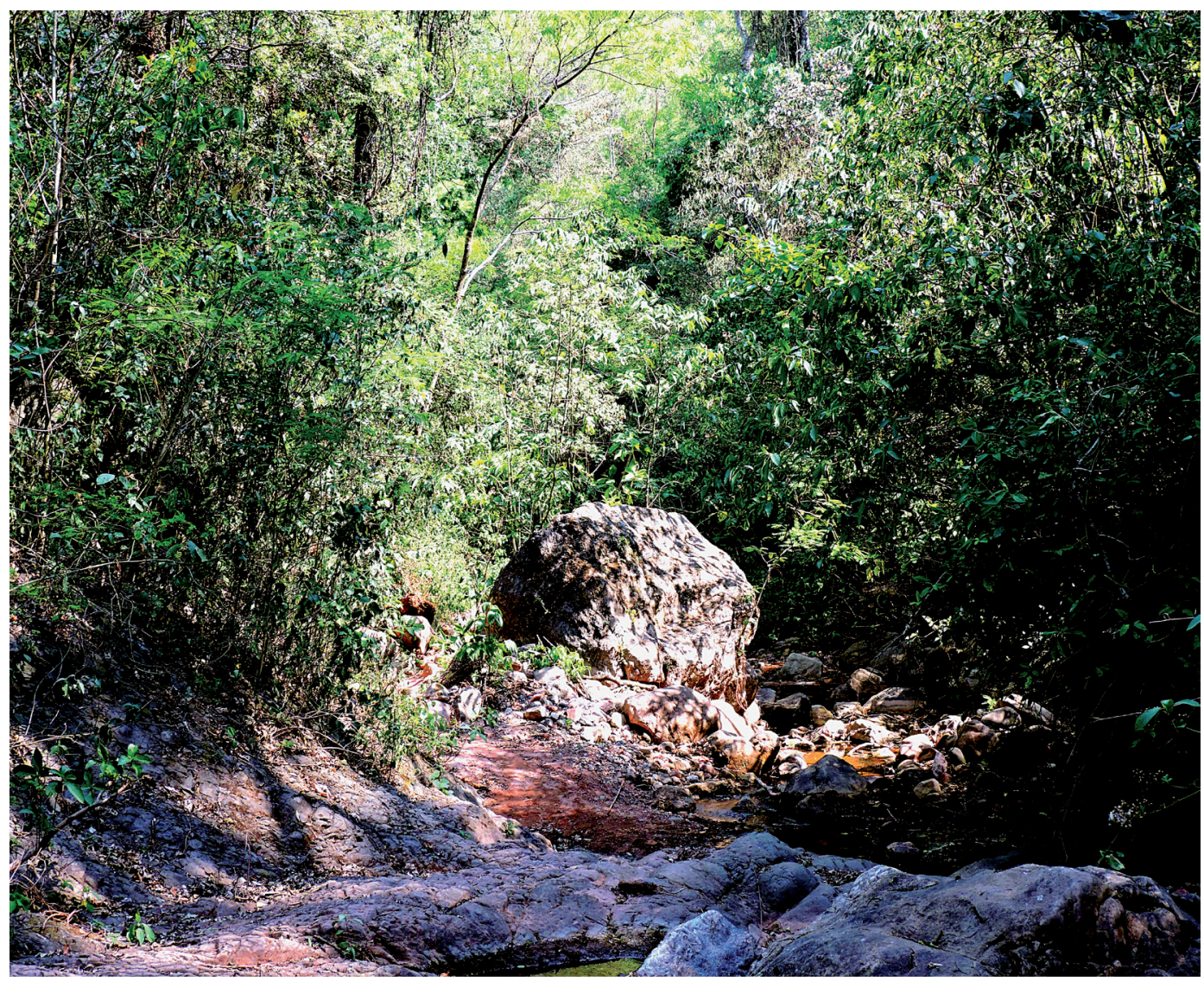

Fig. 2. Arroyo donde se halló el sitio de la congregación. Foto: Ramón Quinteros. $21 / 06 / 2015$. 


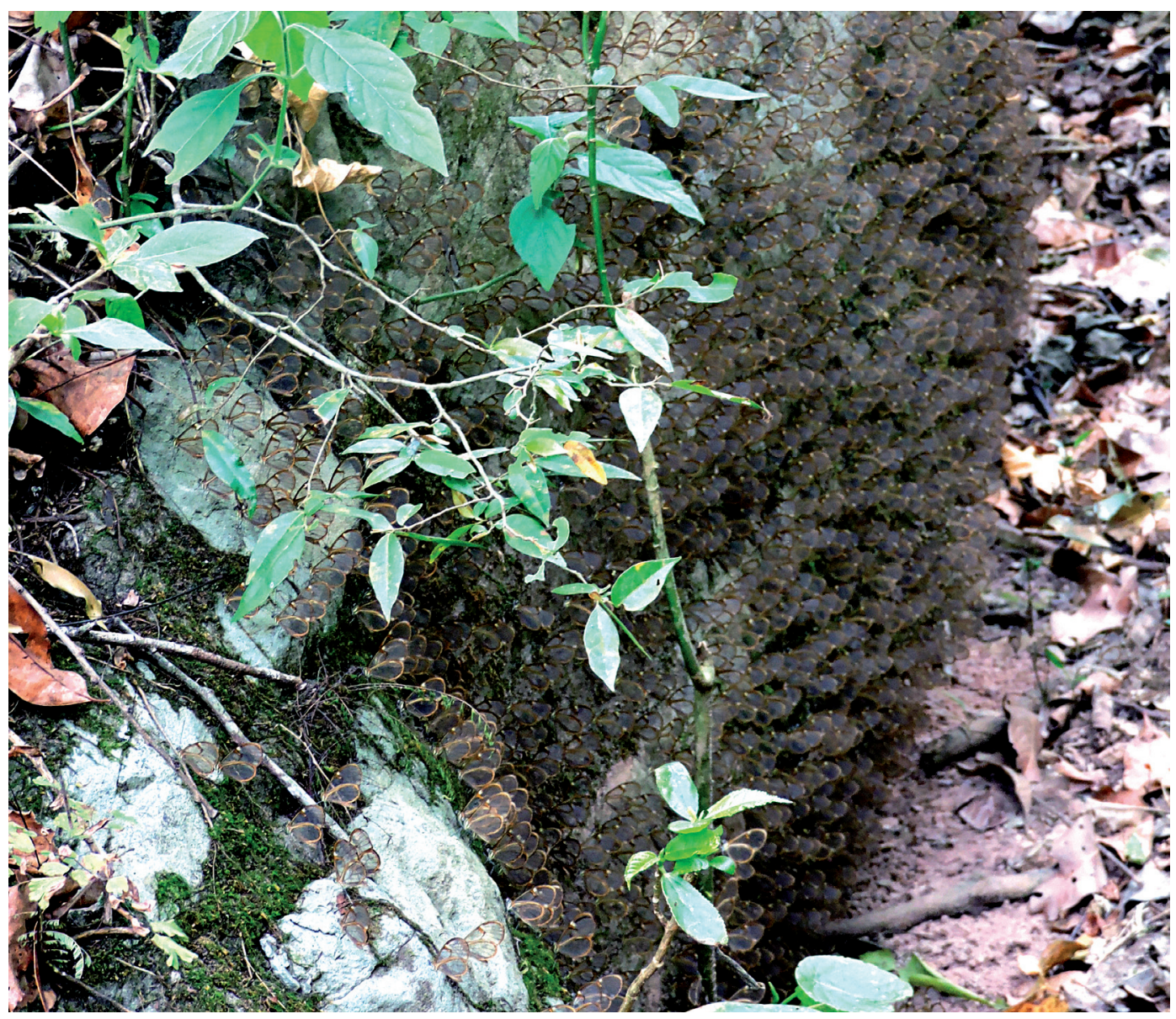

Fig. 3. Gran densidad de individuos sobre una roca del sotobosque. Foto: Ramón Quinteros. $21 / 06 / 2015$.

No se observaron ejemplares alimentándose (salvo fuera del bosque, en unas flores al costado del camino en horas del mediodía) ni siendo vistas predadas por aves u otro animal, así como tampoco ejemplares en cópula. También se vieron en el mismo sitio el 30/8/2014, el 21/6/2015 (sobre la ladera oriental), el 9/7/2016, el 2/9/2017, 9/9/2017, 23/9/2017 y 10/10/2017 (los últimos cinco en la ladera occidental). En los últimos años la cantidad se ha ido incrementando (o es posible sean ahora más visibles sus poblaciones). RQ calcula su número en no menos de 10.000 ejemplares.

En la última fotografía se percibe, entre los muchos Episcada hymenaea, un ejemplar aislado de Pteronymia ozia tanampaya (Fig.
4), la cual es una especie de mayor tamaño y diseño más marcado, y en Argentina es típica del noroeste argentino (Hayward, 1973).

En el año 2017, ya finalizado el invierno, se estableció a $770 \mathrm{msnm}$ en un trecho de $100 \mathrm{~m}$, el cual se halla a escasos $200 \mathrm{~m}$. de la primera cascada de las siete que existen. Este año está en la base de la segunda cascada a exactamente $800 \mathrm{~m}$.

Por ese lugar pasa el único sendero que utilizan gran cantidad de personas que, en los días de intenso calor tratan de llegar a la primera cascada. Cuando hacen contacto con la congregación, puede observarse un gran espectáculo del vuelo en conjunto de miles de estas mariposas. Es éste el primer trabajo donde se reporta y documenta un hábito de 


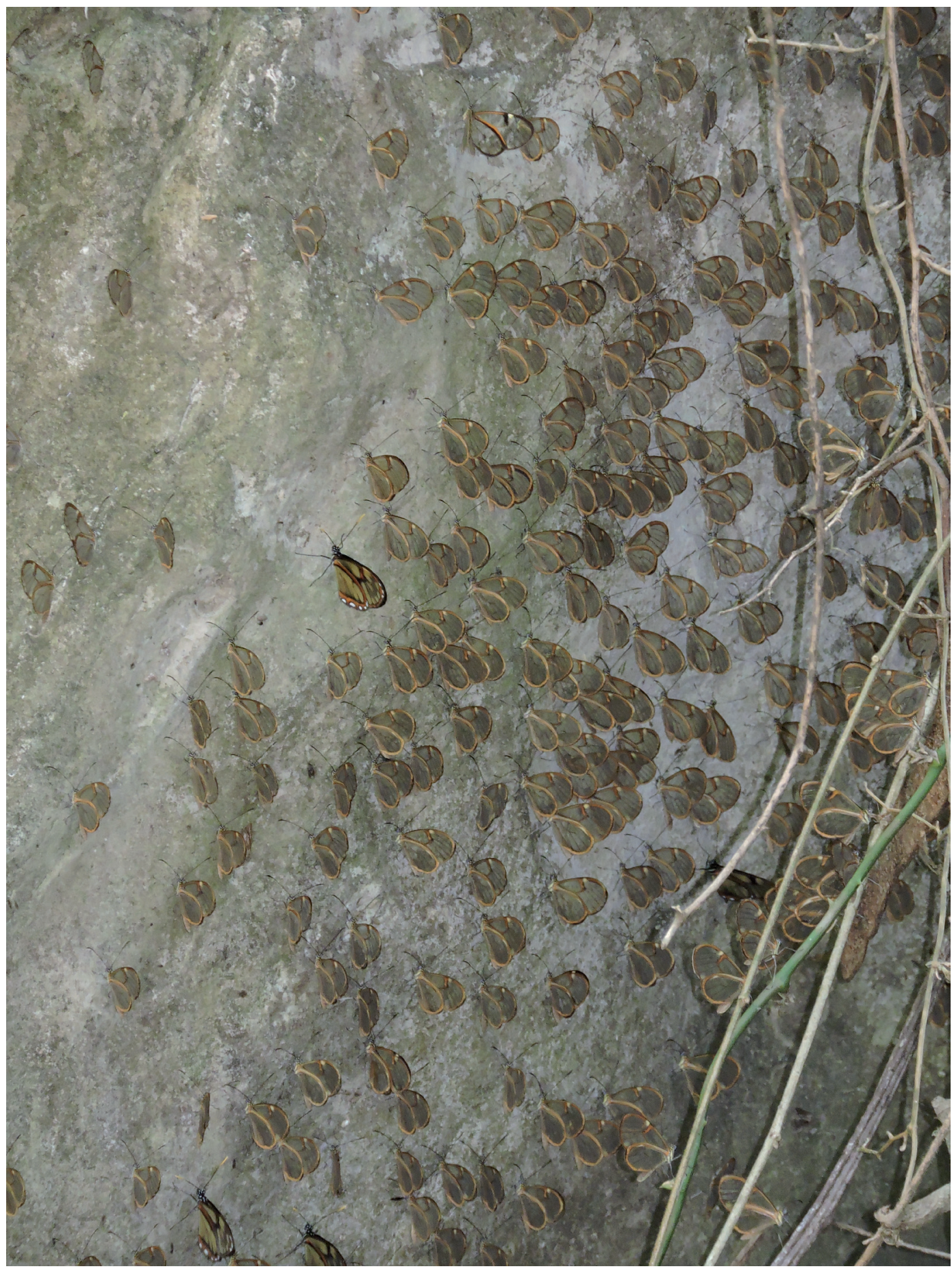

Fig. 4. Casi en el centro de la foto se observa con claridad a Pteronymia ozia tanampaya mezclada con ejemplares de Episcada hymenaea. Foto: Ramón Quinteros. 21/06/2015. 
tal magnitud en la Argentina. Hay subidos en la web tres videos en la siguiente dirección de YouTube: https://www.youtube.com/ results?search_query=Episcada + hymenaea

\section{DISCUSIÓN}

El segundo autor ya había observado anteriormente congregaciones invernales de esta especie en el interior boscoso del Jardín Botánico de la Fundación Miguel Lillo, Tucumán, así como también en cercanías del río Popayán, Parque Nacional El Rey, Salta (unos $300 \mathrm{kms}$ al sur de Salvador Mazza) y en inmediaciones de la ruta nacional 101 (si bien ésta compuesta de varias especies de Ithomiini), dentro del Parque Nacional Iguazú, Misiones (Núñez Bustos, 2009), pero nunca a la escala de la congregación documentada en esta nota. Se trata de un fenómeno poco visto normalmente y que puede pasar desapercibido por su ubicación a menudo dentro de bosques o serranías de poco o difícil acceso.

Evidentemente el intenso calor y la sequía desde 2014 en Salvador Mazza produjo una enorme concentración de ejemplares en el interior húmedo del sotobosque durante la estación seca de los últimos años, mucho más de lo normal con respecto a otros años.

El registro geográfico más cercano publicado de una congregación grande de Episcada hymenaea estaba en el centro de Paraguay (Strickman, 1981). Éste último parece haber sido un poco menor en la extensión de la congregación pero coincide en casi todos los aspectos (época seca del año, conducta, etc.) con la que nos ocupa en este trabajo, así como las congregaciones de varias especies de Ithomiini en Goias, Brasil central (Pinheiro, et al., 2008) y similar a la recientemente publicada de Epityches eupompe en São Paulo y Santa Catarina, en el sur de Brasil (Shirai, Mota, Freitas, 2017), si bien en éste último caso la misma fue hallada en una región húmeda sin estación seca, por lo que se deduce fue el frío el causante de tal agrupación.

Sería interesante estudiar este comportamiento si es que existe también en los otros
Ithomiini de las yungas argentinas como Pteronymia ozia tanampaya, Methona confusa psamathe, Thyridia psidii ino, Dircenna dero celtina y Mechanitis lysimnia elisa. Ésta última es muy común en las yungas y el segundo autor las ha visto volando en gran número (abril de 2015) con dirección hacia el norte en la localidad de Libertador General San Martín y alrededores (Departamento Ledesma, Provincia de Jujuy) aunque no se dispone aún de mayores observaciones.

\section{AGRADECIMIENTOS}

A Fernando Penco por darme a conocer uno de los videos filmado por el primer autor. A André V. L. Freitas (Campinas, São Paulo, Brasil) por la revisión del trabajo y sus sugerencias. A Leonel Baldoni por su ayuda en la edición de algunas fotos.

\section{FINANCIAMIENTO}

Este trabajo no contó con financiamiento institucional.

\section{PARTICIPACIÓN}

RQ participó en el trabajo de campo y aportó los datos. ENB redactó el manuscrito.

\section{CONFLICTOS DE INTERÉS}

Los autores declaran que no existen conflictos de intereses entre ellos o con terceras personas.

\section{LITERATURA CITADA}

Brown, K. S., Vasconcellos-Neto, J. (1976). Predation on aposematic ithomiine butterflies by tanagers (Pipraeidea melanonota). Biotropica 8 (2): 136141

Freitas, A. V. L., Vasconcellos-Neto, J., Vanini, F., Trigo, J. R., Brown, K. S., (2001). Population studies of Aeria olena and Tithorea harmonia (Nymphalidae, Ithomiinae) in Southeastern Brazil. Journal of the Lepidopterists' Society 55: $150-157$

Hayward, K. J. (1973). Catálogo de los ropalóceros argentinos. Opera Lilloana, 23: 1-318.

Núñez Bustos, E. (2009). Mariposas diurnas (Lepidoptera: Papilionoidea y Hesperioi- 
dea) del Parque Nacional Iguazú, Provincia de Misiones, Argentina. Tropical Lepidoptera Research, 19 (2): 71-81.

Núñez Bustos, E. (2010). Mariposas de la Ciudad de Buenos Aires y alrededores. Buenos Aires, Vázquez Mazzini Editores.

Pinheiro, C. E. G., Medri, I. M., Salcedo, A. K. M. (2008). Why do ithomiines (Lepidoptera, Nymphalidae) aggregate? Notes on a butterfly pocket in central Brazil. Revista Brasileira de Entomologia, 52: 610-614.
Shirai, L. T., Mota, L. L, Freitas, A. V. L. (2017). Aggregation of Epityches eupompe (Nymphalidae: Ithomiini) in southern Brazil. Tropical Lepidoptera Research, 27 (2): 111-114.

Strickman, D. (1981). Observation on an aggregation of Episcada hymenaea Prittev. [sic] (Ithomiidae) in Paraguay. Journal of the Lepidopterists' Society, 35 (2): 158.

Volkmann, L., Núñez Bustos, E. (2013). Mariposas Serranas. Guía de especies más comunes halladas en sierras, valles y salinas del centro oeste argentino. Tomo II. Nymphalidae y Hesperiidae. Huerta Grande, Equipo Gráfico. 